\title{
La Factoría Cultural y la Marca Niemeyer. Avilés: una ciudad creativa en el Arco Atlántico ${ }^{1}$
}

\author{
Natalia Tielve García \\ Universidad de Oviedo \\ tielvega@uniovi.es
}

RESUMEN: El Centro Niemeyer y la Factoría Cultural constituyen los dos ejes estratégicos sobre los que se ha asentado el proyecto de revitalización urbana y dinamización socioeconómica promovido por la ciudad de Avilés que se examina en este trabajo. Con el propósito de transformarse en una de las ciudades creativas del Arco Atlántico, en Avilés se ha fraguado una nueva identidad, una marca ligada a la innovación, la modernidad, la cultura y el arte que ha tenido un positivo impacto en el desarrollo del sector servicios, la promoción de las industrias culturales y el avance de la producción tecnológica.

PALABRAS CLAVE: Ciudades creativas; Centro Niemeyer; Factoría Cultural; Avilés; Arco Atlántico; Reconversión urbana.

\section{Cultural Factory and Niemeyer's Brand. Avilés: a Creative City in the Atlantic Arc}

ABSTRACT: The Niemeyer Centre and the Factoría Cultural (Cultural Factory) constitute the two strategic axes serving as the cornerstones for the urban revitalization and socioeconomic dynamization project fostered by the city of Avilés, analyzed in this paper. With the purpose of becoming one of the creative cities in the Atlantic Arc, a new identity has been forged in Avilés, a brand pledged to innovation, modernity, culture and the arts, which has had a positive effect in the development of the service sector, the promotion of cultural industries and the progress of technological production.

KEYWORDS: Creative Cities; Niemeyer Centre; Factoría Cultural; Avilés; Atlantic Arc; Urban Restructuring.

Recibido: 9 de febrero de 2020 / Aceptado: 16 de junio de 2020.

\section{La construcción del nuevo Avilés: marketing urbano e ingeniería cultural}

Si uno de los rasgos caracterizadores de la sociedad postindustrial es la apuesta por una economía basada en las industrias culturales y los servicios, la promoción de la innovación y la creación de empresas de alto nivel tecnológico (Bell, 1989: 64) esta es la dinámica en la que la ciudad de Avilés, tras experimentar las consecuencias de los procesos de reestructuración económica y el declive industrial, ha querido abrirse camino en las últimas décadas². Con el objetivo de transformarse en una de las ciudades creativas del Arco Atlántico (Scott, 2006: 2), Avilés ha impulsado un ambicioso proyecto estratégico que cuenta como soportes esenciales con dos nuevos equipamientos: el Centro Niemeyer y la Factoría Cultural. Símbolos del recambio, con ellos se ha forjado una nueva imagen y una renovada forma de hacer ciudad fundamentada en la innovación y en la cultura ${ }^{3}$.

Ligada al Plan Estratégico Avilés 2000 y, de modo preferente, al Plan General de Ordenación del Término Municipal de Avilés ${ }^{4}$ redactado por el arquitecto Eduardo Leira ${ }^{5}$ en 2006 (Leira y Calvo, 2002-2003: 103-128), la reconversión urbana emprendida se ha sustentado en una fuerte inversión económica. La financiación, en buena medida derivada de fondos europeos procedentes del FEDER y las acciones Urban, Interreg, IFOP y Equal, ha permitido afrontar la regeneración de la ría

Cómo citar este artículo: TIELVE GARCÍA, Natalia, «La Factoría Cultural y la Marca Niemeyer. Avilés: una ciudad creativa en el Arco Atlántico», Boletín de Arte-UMA, n. 41 , Departamento de Historia del Arte, Universidad de Málaga, 2020, pp. 239-250, ISSN: 0211-8483, e-ISSN: 2695-415X, DOI: http://dx.doi.org/10.24310/BoLArte.2020. v41i.7778 


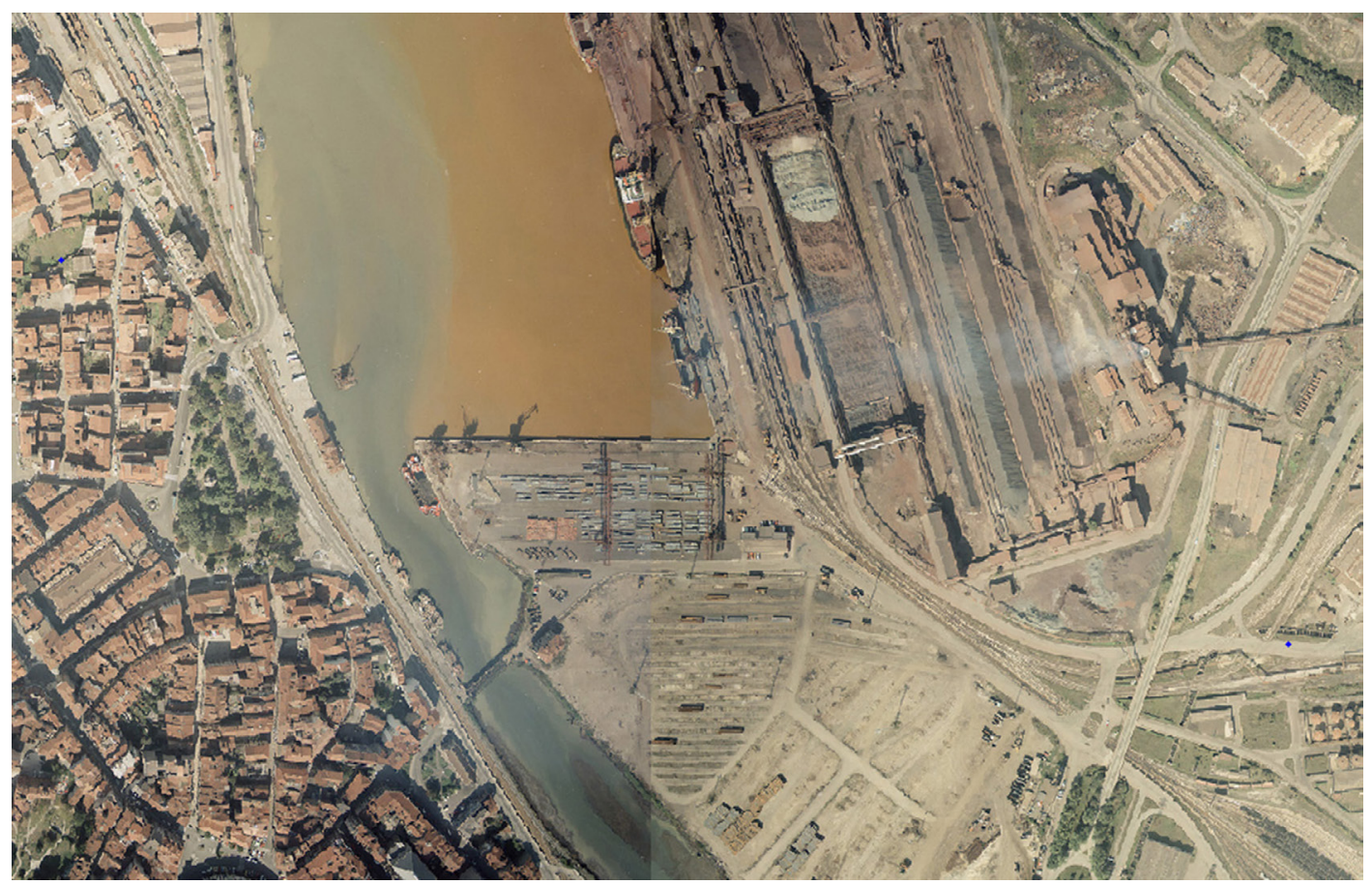

1. Instituto Geográfico Nacional, Vuelo 1989-1991, Costas. Fotograma en color a escala 1:5.000. La imagen muestra el fondo de saco de la ría de Avilés, con la dársena de San Agustín y los muelles vinculados a la actividad siderúrgica. Fuente: Fototeca digital. Centro Nacional de Información Geográfica

y los espacios portuarios (Menéndez, 2014), la rehabilitación del casco histórico y la implantación de una cultura basada en la I+D+i

Una de las apuestas fundamentales de esta planificación es la creación del área de nueva centralidad en la que adquiere un papel determinante la recuperación y la reintegración en la trama urbana del frente marítimo, siguiendo planteamientos inspirados en las llamadas ciudades del agua (Alemany, 2015: 76-79). El modelo de Nantes ha resultado en este sentido singularmente aleccionador: la posibilidad de generar una nueva centralidad a partir de una isla, natural en Nantes y artificial en Avilés, intervenir sobre el espacio público, crear nuevas dotaciones -en particular asociadas al arte, la cultura y el ocio- y generar una nueva identidad. En efecto, en la ciudad-región de Nantes, al objeto de hacer frente a las consecuencias de la desindustrialización, comenzaba a cobrar forma en la década de 1990 un proceso de regeneración con el que se ha procurado armonizar la preservación del patrimonio con la revitalización urbana (Masboungi, 2003). En el marco de este proceso, guiado por la máxima de la planificación flexible, se inscribe la actuación pergeñada y desarrollada por los arquitectos urbanistas, especialistas en paisaje, Alexandre Chemetoff y Jean Louis Berthomieu: el Plan-guide de île de Nantes. Desde la premisa de construir la ciudad sobre sí misma, el planeamiento, sujeto a una constante reformulación, prima la renovación sobre el crecimiento en extensión y mantiene como criterio básico la pervivencia de la memoria industrial.

Siguiendo, pretendidamente, el modelo de Nantes, en Avilés se ha orquestado un programa que comprende un conjunto de actuaciones encabezadas por La Isla de la Innovación (Fundación Metrópoli, 2008). Planteada en 2008 con el propósito de regenerar y dinamizar el entorno degradado de la ría tras décadas de actividad industrial [1] ${ }^{7}$, en su diseño se han conjugado diversas propuestas que, entre otros elementos, incluyen la creación de un espacio singular presidido por el Centro Niemeyer ${ }^{8}$. El estuario avilesino, con la nueva planificación, deja de funcionar como barrera entre el espacio de producción y el de residencia para pasar a convertirse en nexo de unión [2]. De este modo, la mayoría de 
2. Vuelo fotogramétrico del

Plan Nacional de Ortofotografia Aérea (PNOA) del año 2017. Escala 1: 25.000. La imagen muestra la misma zona que la anterior, evidenciando la trasformación experimentada tras el desmantelamiento de las instalaciones industriales y con la erección del Centro Niemeyer. Fuente: Fototeca digital. Centro Nacional de Información Geográfica

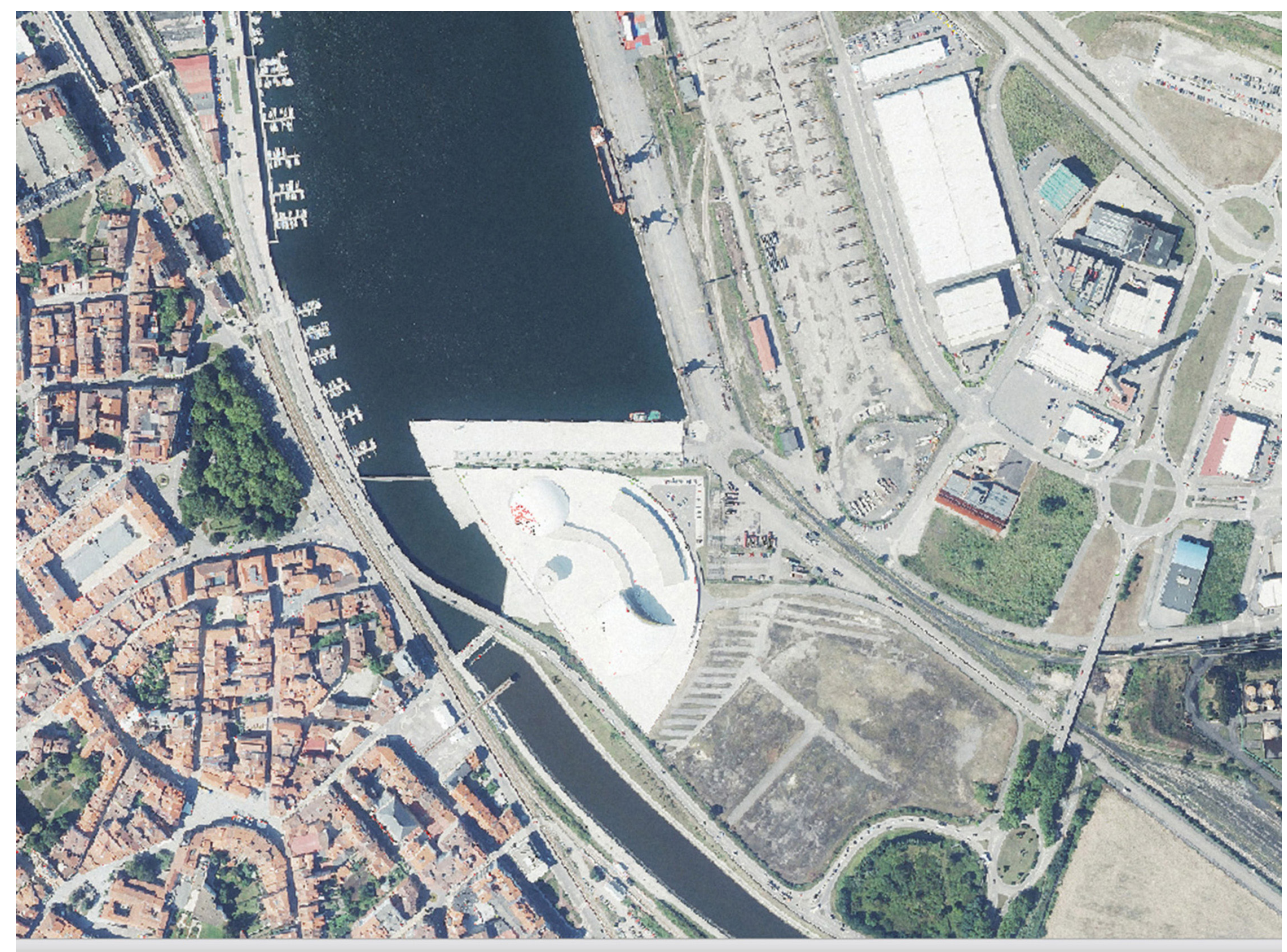

las intervenciones proyectadas se centran en el puerto -la terminal de cruceros, el paseo y la nueva marina, entendida como un espacio reservado a las actividades deportivas y recreativas- y el resto de las actuaciones planteadas, aunque pendientes de realización, están vinculadas a la creación del bulevar, una gran avenida arbolada concebida como el principal acceso urbano y eje de comunicación entre el casco histórico y el Centro Niemeyer, el parque de los equipamientos, con instalaciones deportivas y de ocio, la plaza de la estación, con la reestructuración de la red de vías y la construcción de un intercambiador multimodal, el salón del ferrocarril, vinculado a la integración ferroviaria y los llamados Cubos de la Innovación, edificios eco-tecnológicos donde se combinan servicios de restauración, espacios expositivos y oficinas turísticas.

\section{La marca Niemeyer y la memoria de la industria}

Partiendo del proyecto elaborado por el arquitecto Oscar Niemeyer, tras un intrincado proceso, el Centro Niemeyer abría sus puertas en el año 2011. Galardonado con el Pre- mio Príncipe de Asturias de las Artes en 1989, el arquitecto brasileño ${ }^{9}$ hizo entrega de un diseño a la Fundación Príncipe de Asturias, con motivo de su XXV aniversario, para la creación de un espacio museístico dedicado a los Premios (Bermejo y Álvarez, 2018: 75-80). Aunque la propuesta no salió adelante, lo cierto es que a partir de esta fue elaborado un anteproyecto que constituye el embrión del centro cultural (Consejería de Cultura y Turismo del Principado de Asturias, 2010).

Con una cuantiosa inversión que ronda los cuarenta y tres millones de euros, el equipamiento fue desde muy pronto presentado como una oportunidad para la mejora, el desarrollo económico y la internacionalización de la ciudad de Avilés (Tielve, 2018: 151-158). El efecto Niemeyer, esto es, la sinergia mediática y socioeconómica que, pretendidamente, debía propiciar el nuevo equipamiento, tenía como referente inmediato el impacto alcanzado por el Museo Guggenheim, motor y símbolo del proceso de transformación, reurbanización y de cambio de imagen experimentados por la ciudad de Bilbao ${ }^{10}$. Como es conocido, con la apertura del Museo Guggenheim fueron orquestadas unas políticas de promoción económica basadas en el potencial cultural y turístico 


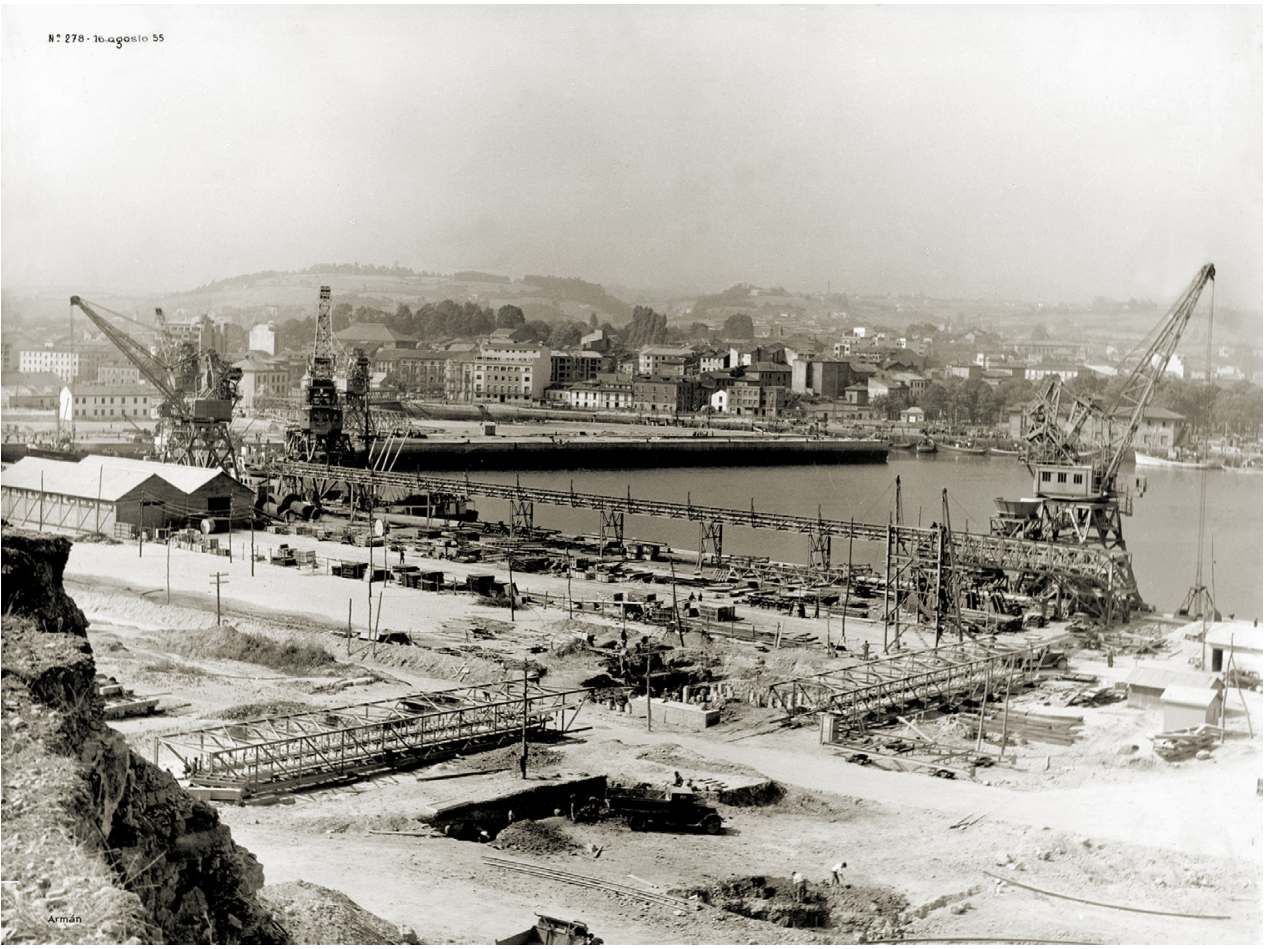

3. Vista parcial del puerto de Avilés, con los muelles siderúrgicos de Ensidesa en construcción. Agosto 1955. Colección Armán. Fuente: Archivo Histórico de Asturias. Fondo documental de Ensidesa de Bilbao pero, paralelamente, se emprendieron actuaciones de gran calado que llevaron a la pérdida de una parte muy significativa de su patrimonio industrial. El traslado de la actividad portuaria desde Abandoibarra a Santurtzi permitió ganar terreno a la ría y liberar una gran franja de suelo muy atractiva por su localización y extensión -unos 30.000 metros cuadrados- para nuevos usos. Así, en los terrenos de los desmantelados Astilleros Euskalduna, se construía a finales de la década de 1990 el Palacio de Congresos y de la Música. Del mismo modo, bajo el Puente Euskalduna, a partir de 2001, se abría el Museo Marítimo en recuerdo de la otrora actividad portuaria e industrial.

En consonancia con la capital vizcaína, Avilés es una ciudad con un pasado reciente marcado por la industrialización, la reestructuración económica, la crisis y la reconversión [3]. De ahí que Bilbao haya sido el espejo en el que mirarse, en un intento de reconstruir Avilés, modificar su modelo productivo e introducir un giro hacia los servicios, la innovación y la producción tecnológica. Siguiendo el modelo bilbaíno, en Avilés no solo se ha proyectado y construido un gran complejo cultural -una arquitectura espectacular, avalada por una firma de prestigio- sino que además, en torno a este, se ha diseñado una marca ligada al diseño, al arte, a la cultura y al desarrollo; un marca que, convenientemente gestionada a nivel mediático, está llamada a conquistar una mayor presencia internacional [4]. Así, al igual que en Bilbao, la conquista de esa nueva posición, de esa renovada imagen y de esa proyección internacional no se ha hecho a coste cero y las huellas del pasado industrial, nuevamente, se han ido difuminando ${ }^{11}$. Buena parte de las instalaciones fabriles históricas se han desmantelado y, en el mejor de los casos, se han planteado para ellas intervenciones encaminadas a reconvertirlas en nuevas dotaciones, con frecuencia en detrimento de sus valores técnicos e identitarios (Suárez, 2013: 208-209).

En este escenario, el Centro Niemeyer constituye la pieza central de un producto turístico basado en la promoción cultural y la creatividad [5]. Bautizado como un contenedor de ilusiones, el nuevo equipamiento comenzaba a generar actividad ya en los años previos a su inauguración. Una macrocampaña publicitaria impulsada desde 2007 estuvo destinada a propagar el previsible impacto económico, urbanístico, turístico y cultural que el nuevo espacio iba a lo$\operatorname{grar}^{12}$. Incluía un amplio programa de actividades desarrolla- 
das en Cannes, Madrid, Brasilia y Shanghái, junto con otras capitales, contando con la colaboración de Woody Allen, Paco de Lucía, Carlos Saura, Paulo Coelho y Win Wenders, entre otros. En esta línea se estrechaban los lazos, cuando menos en el plano mediático, entre el Centro Niemeyer, el Museo Guggenheim de Bilbao y, más adelante, el Centro Botín de Santander, aspirando a crear un eje sustentado en un circuito de museos e infraestructuras culturales [6]. Paralelamente, a nivel autonómico se creaba un nuevo producto cultural, conocido como Pentágono del Arte, presentado oficialmente en la Feria Internacional de Arte Contemporáneo de Madrid, ARCO, en 2017 y constituido por cinco equipamiento artísticos: LABoral, Centro de Arte y Creación Industrial, Museo de Bellas Artes de Asturias, Sala Borrón, Museo Barjola y Centro Niemeyer.

Esta intensa actividad, prolongada durante los primeros años de andadura de la institución, derivó en una complicada situación económica que, en la nueva etapa del centro -con un paréntesis de cierre no exento de polémica y un concurso de acreedores- ha tratado de reconducirse en aras de alcanzar un equilibrio presupuestario. De hecho, tras unos prometedores primeros pasos, las dudas sobre el modelo de gestión del equipamiento, las desavenencias entre la administración regional, la local y la fundación que gestiona el centro

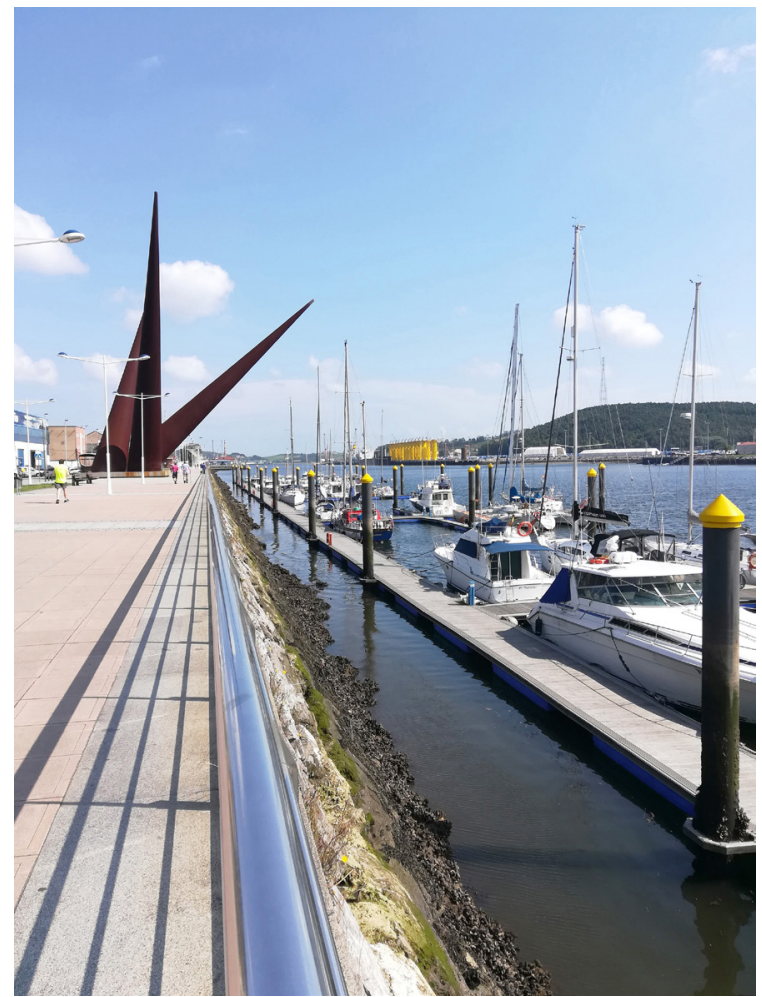

4. La escultura Avilés, de Benjamín Menéndez, inaugurada en 2005, es una de las intervenciones artísticas que han contribuido a la recuperación del paseo de la ría de Avilés (fotografía: NTG)
5. Vista parcial del Centro Niemeyer, inaugurado en 2011, con la torre mirador en primer plano y el auditorio al fondo (fotografía de la autora)

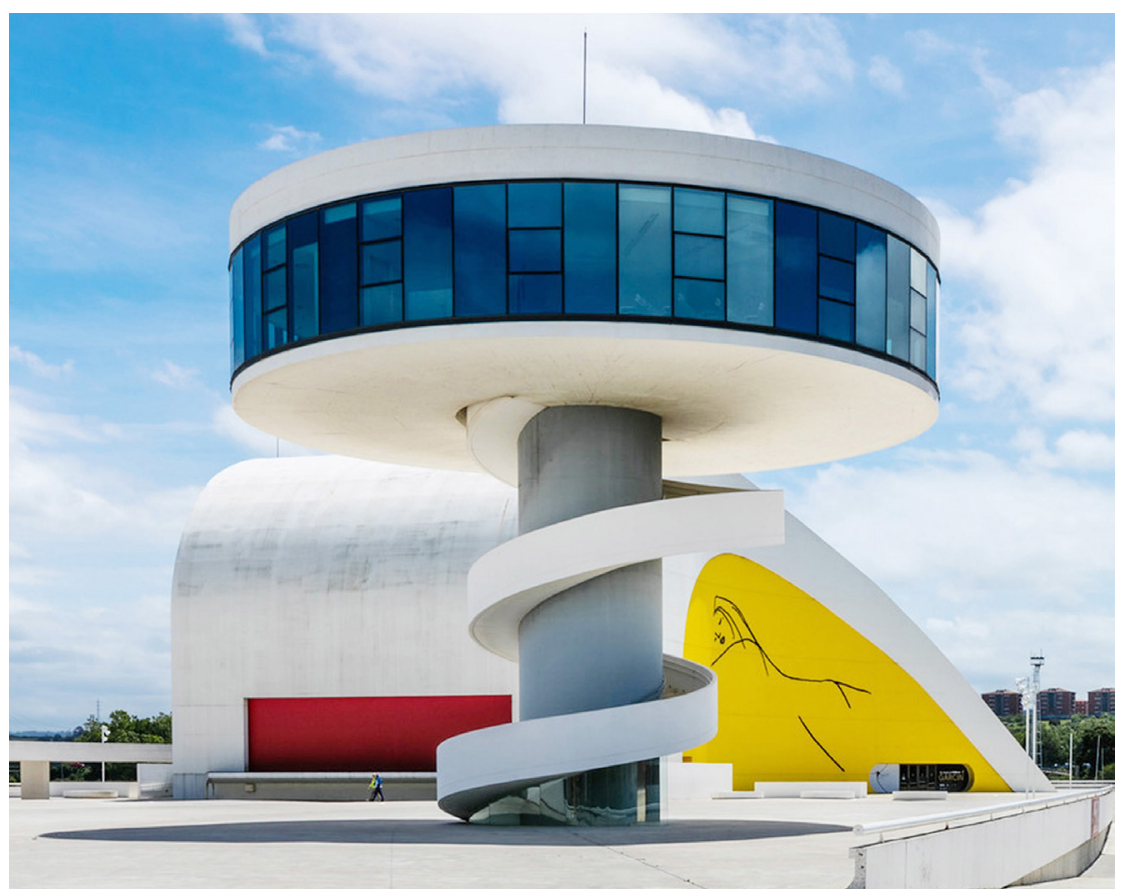




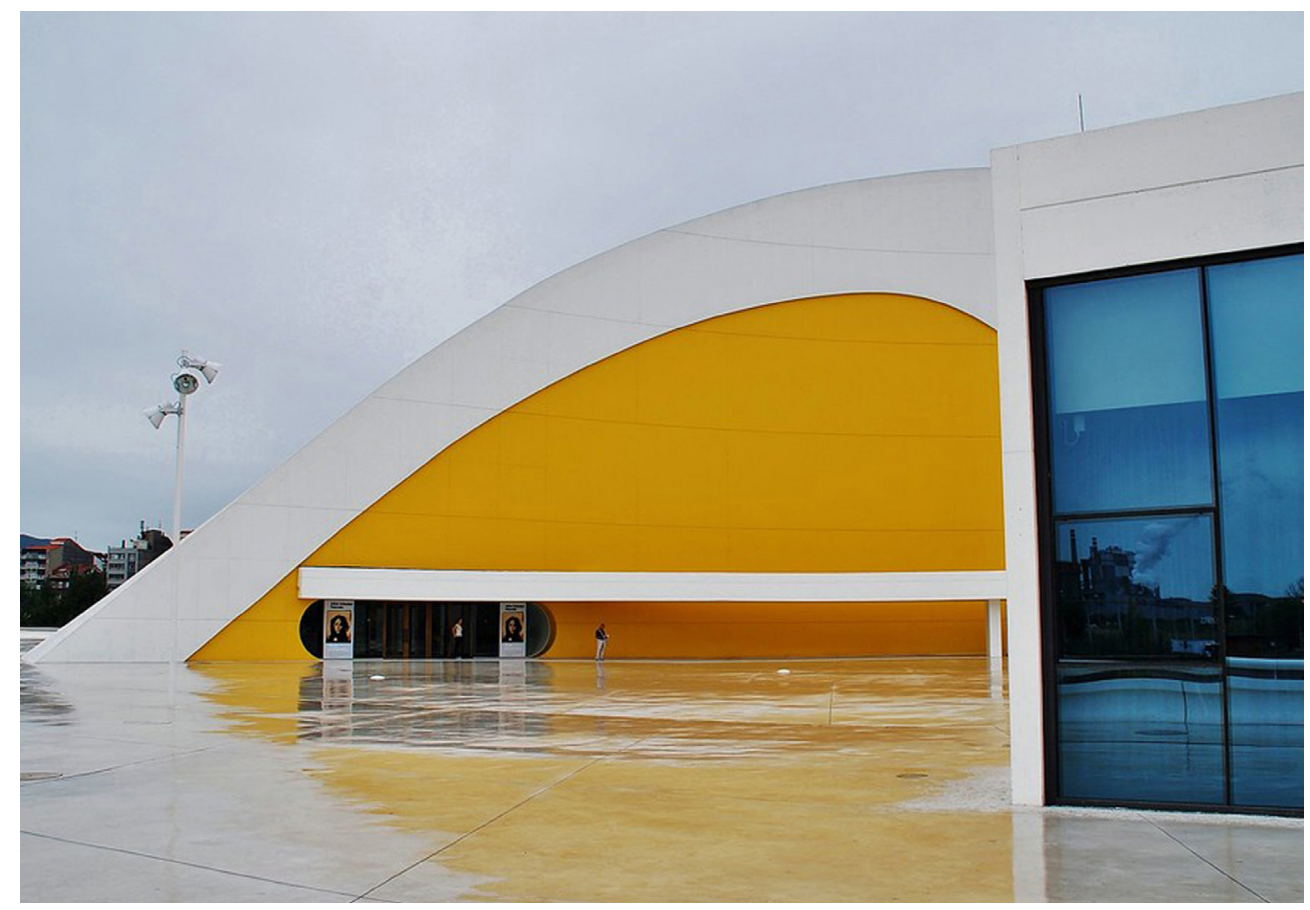

6. Vista parcial del equipamiento cultural. La marca Niemeyer se ha convertido en símbolo de la regeneración urbana emprendida en Avilés (fotografía de la autora)

han puesto en tela de juicio la viabilidad del proyecto [7]. No obstante, cierto es que, con su repercusión mediática, el nuevo complejo cultural ha dotado de visibilidad a la ciudad de Avilés. Ha proyectado una imagen de marca fundamentada en la modernidad, la cultura, la creatividad y la innovación, alcanzando un efecto positivo en la promoción de la economía local y en el desarrollo del sector servicios ${ }^{13}$. Teniendo como eje de atención el Centro Niemeyer, la oferta turística de la ciudad ha experimentado un crecimiento notable, se han diseñado políticas de marketing vinculadas a redes como Spanish Convention Bureau y Cool Cities, se ha incrementado la oferta cultural y se han renovado los servicios y equipamientos públicos. En esta senda se inscribe el proyecto de la Factoría Cultural del que a continuación nos ocuparemos.

\section{La Factoría Cultural: creatividad, innovación y producción}

El Libro Blanco Crecimiento, competitividad, empleo. Retos y pistas para entrar en el siglo XXI (Comunidades Europeas,
1993), exploraba el potencial de los nuevos yacimientos de empleo basados en factores como la creatividad, la innovación y la producción. A partir de este modelo, las nuevas demandas ciudadanas relacionadas con las actividades de cultura y de ocio han sido abordadas desde diversos enfoques estratégicos, incluyendo aspectos como la creación cultural, el desarrollo local, la revalorización de los espacios públicos y del patrimonio cultural, el turismo, las nuevas tecnologías de la información y las actividades deportivas y recreativas.

En el caso de nuestro país, los Planes de Fomento de Industrias Culturales que desde el año 2009 se vienen impulsando han apostado por la formación, la especialización, el desarrollo y la internacionalización de estas industrias, financiando proyectos mediante subvenciones directas, con programas de ayudas reembolsables, ayudas complementarias y avales, además de la dotación de becas y premios. Siguiendo esta línea, el Libro Blanco sobre Industrias Creativas en el Principado de Asturias planteaba la oportunidad de realizar una «radiografía precisa de las distintas actividades que conforman la cultura como sector económico» (Con- 


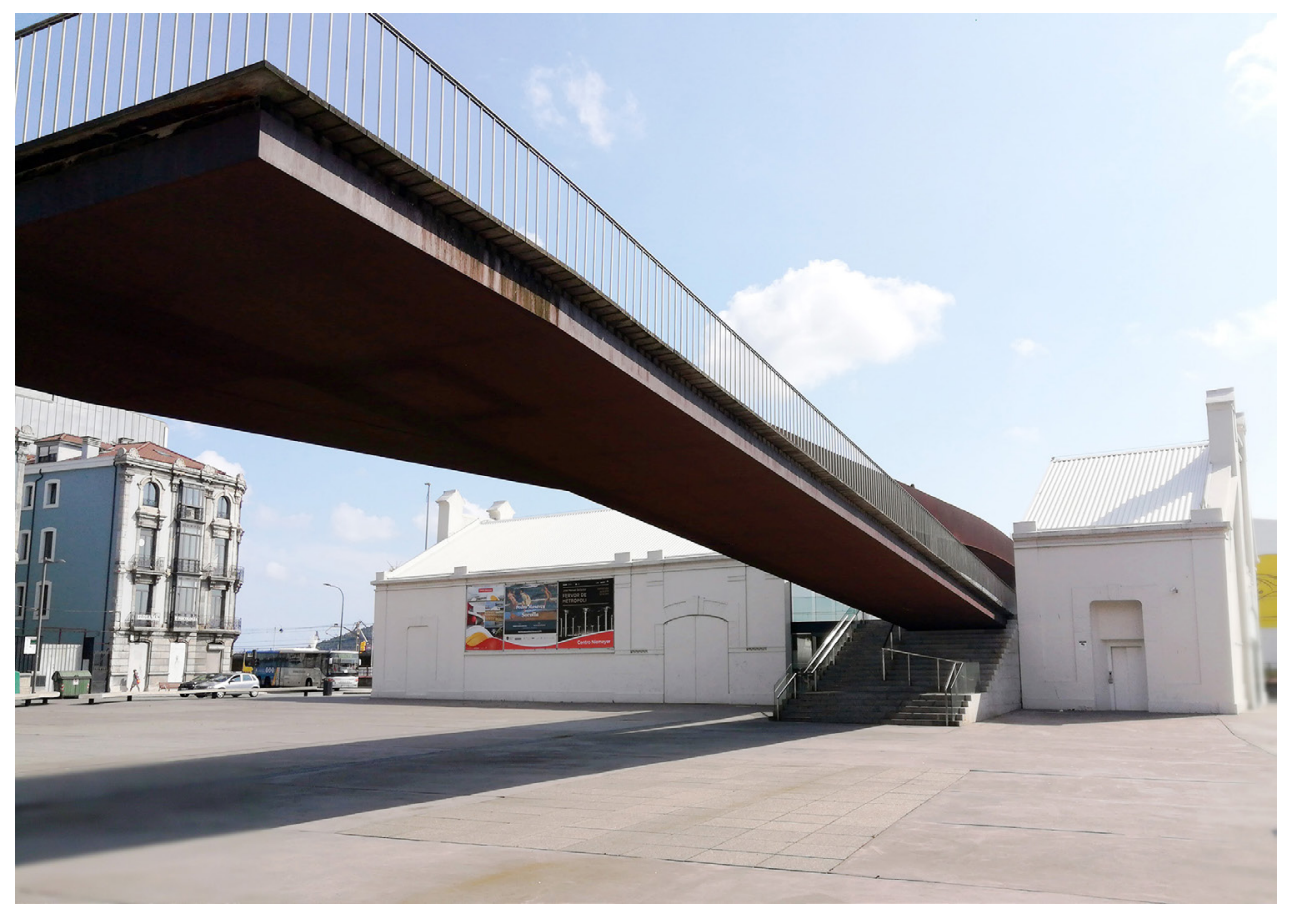

7. La antigua pescadería municipal de Avilés, obra del arquitecto Antonio Alonso Jorge, fechada en 1916, seccionada para dar cobijo a la pasarela peatonal que conduce al Centro Niemeyer (fotografía de la autora)

sejería de Cultura y Turismo, 2009: 3) abarcando sectores como el editorial, el audiovisual, el musical, las artes escénicas, las artes visuales y los videojuegos.

En el ámbito de las industrias creativas se inserta una serie de equipamientos que en los últimos años se ha impulsado en la ciudad de Avilés. A su cabeza se sitúa la Factoría Cultural, una incubadora de proyectos creativos que reúne un conjunto de actividades relacionadas con el arte y la cultura que van desde la formación a la difusión, pasando por la producción cultural. Concebida como un espacio de carácter multidisciplinar, se sitúa en el complejo que ocupaba la firma Confecciones Camino S. A., históricamente dedicada a la fabricación de camisas ${ }^{14}$. La fábrica, en desuso desde diciembre de 1995, mediante convenio urbanístico pasó a manos del ayuntamiento que llevó adelante su remodelación [8].

La reconversión de la antigua factoría, en todo caso, ha de contemplarse en el marco de una serie de actuaciones llevadas a cabo en la ciudad en los últimos años [9]. Es el caso de las emprendidas en las antiguas instalaciones de la curtidora Avilés Industrial, la fábrica de harinas de ce- real El Águila, el astillero Fernández Hevia o la pescadería municipal que, muy dispares entre sí en lo que a criterios de intervención, rehabilitación y reconversión se refiere, han dado lugar a nuevos equipamientos (Tielve, 2016: 93-94). Ahora bien, como previamente apuntábamos, con frecuencia los edificios se han transformado en simples contenedores despersonalizados, privados de su identidad y vacíos de contenido.

\section{Fábricas para la creación}

La idea de crear un espacio como la Factoría Cultural, partiendo de la recuperación de una antigua fábrica para dotarla de un nuevo uso ligado a la creación artística ${ }^{15}$, puede relacionarse con otras interesantes experiencias. Es el caso de la desarrollada en Hangar, Centro de investigación y producción artística, que ocupa un edificio originalmente destinado a la producción textil -en la actualidad de titularidad municipal- en el complejo de Can Ricart en Poblenou (Barcelona). Cuenta, como es conocido, con un consolidado programa 


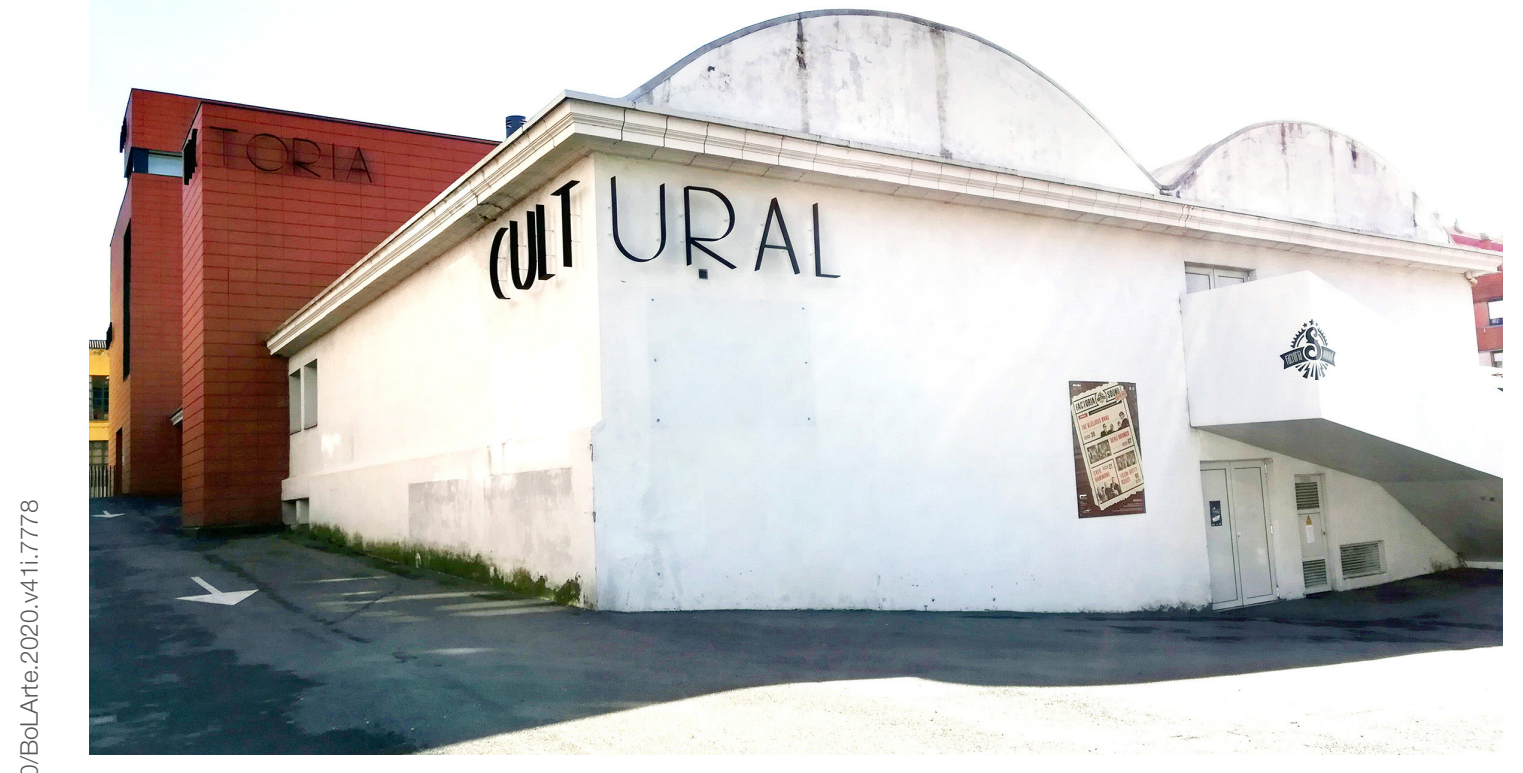

8. La fábrica de camisas Confecciones Camino S. A., erigida en 1946 partiendo de las trazas del arquitecto Tomás Menéndez Abascal, ha sido reconvertida en la Factoría Cultural (fotografía de la autora)

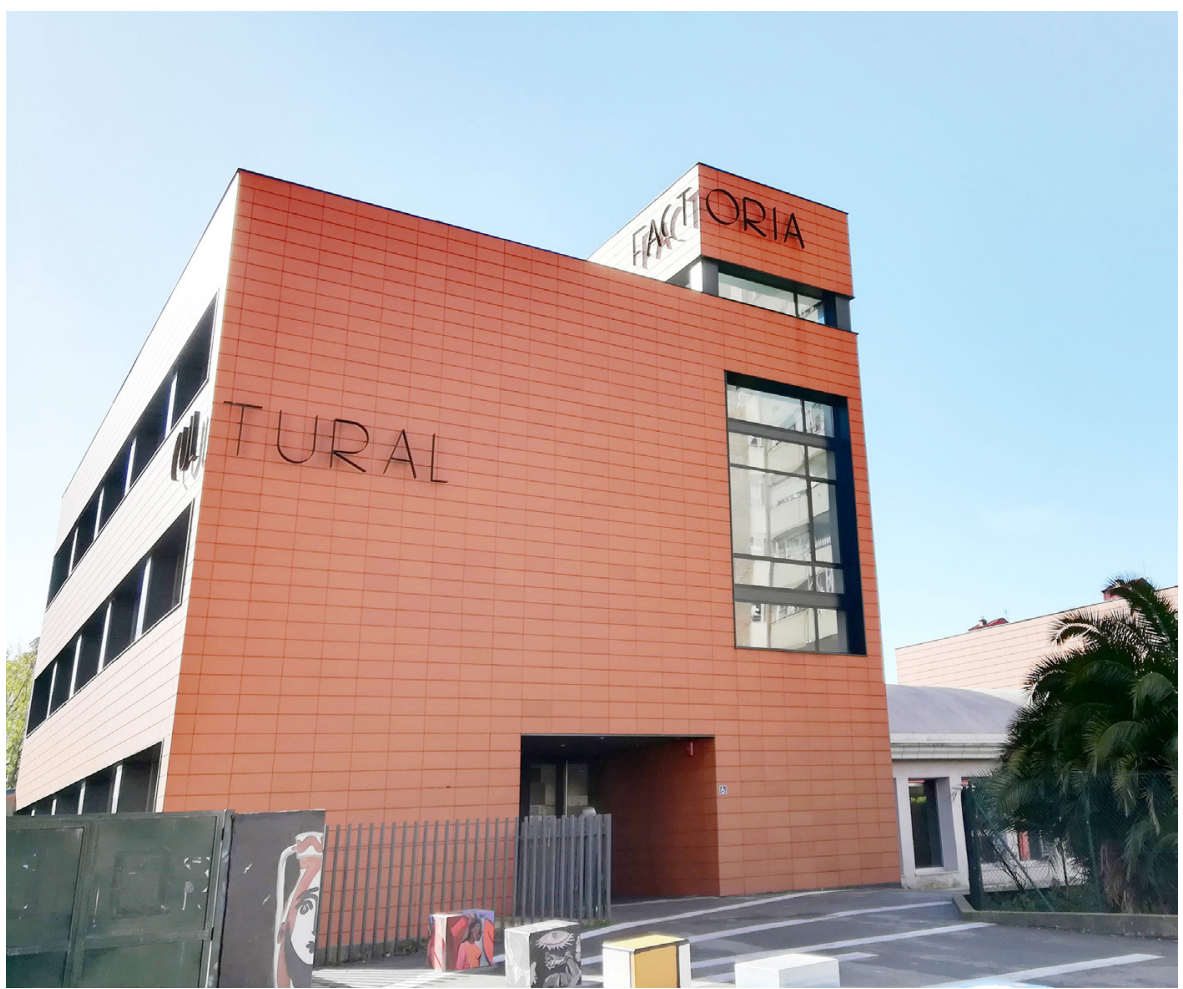

9. La Factoría Cultural ocupa el edificio de la antigua fábrica de camisas, tras la actuación llevada a cabo entre los años 2009 y 2012, siguiendo el proyecto del arquitecto Aitor López Galilea (fotografía de la autora) 
de residencias y un laboratorio de arte interactivo de reconocimiento internacional. Puede además relacionarse con un atractivo programa, Fábricas de Creación, arbitrado por el Ayuntamiento de Barcelona con la voluntad de extender la red de equipamientos municipales, vinculados a la creación y la producción cultural a través de la reconversión de recintos fabriles en desuso, con espacios como Fabra i Coats en Sant Andreu, Graner en Sants-Montjuïc, La Seca en Ciutat Vella, La Escocesa en Sant Martí, La Caldera en Les Corts y la Sala Beckett/Obrador en Sant Martí. Al entenderse como incubadoras de creación, son equipamientos que dan cabida a la experimentación, los nuevos medios y las propuestas interdisciplinares, incentivando la política cultural y la apuesta por la creatividad (Estankona, 2013: 29-30).

Con unos presupuestos cercanos, en Bilbao se ha desarrollado desde 2008 el proyecto ZAWP-Zorrotzaurre Art Working Progress, incluido dentro del programa de fábricas de cultura que promueve el Gobierno Vasco (Sarriugarte, 2014: 50-51). Vinculado a la iniciativa de la asociación cultural Hacería Arteak se ha orientado hacia la reconversión de antiguos edificios industriales, localizados en la Ribera de Deusto-Zorrotzaurre, para dar lugar a una isla creativa que incluye actividades como el teatro, la música, la danza, la cultura urbana, la fabricación digital y las industrias creativas. A estos referentes nacionales pueden añadirse otros internacionales, como es el caso, para no extendernos, de ZKM -Zentrum für Kunst und Medientechnologie- de Karlsruhe (Alemania). Emplazado en la región de Baden-Wurtemberg, fundado en 1989, ha contribuido de forma decisiva a la dinamización de una ciudad visiblemente afectada por el desmantelamiento industrial ${ }^{16}$.

\section{Producción, creación e internacionalización}

En el año 2010 comenzaba su andadura la Factoría Cultural, contando con fondos europeos a través del proyecto Innovate ${ }^{17}$ y marcándose como objetivos esenciales el fomento de la industria cultural y creativa, brindar formación, espacios expositivos y talleres para la creación. Con estos ingredientes, el espacio desarrolla diferentes iniciativas que persiguen no solo a dar a conocer la producción que en el centro se genera, sino también mejorar la capacidad de los creadores para acceder al mercado y promover el uso de las nuevas tecnologías y de las redes sociales. De ahí su participación en proyectos europeos vinculados al programa Europa Creativa y Erasmus+. La labor de nuevo equipamiento se concentra en la creación digital y audiovisual, contando con espacios destinados a talleres -cerámica, pintura, encuadernación, marroquinería, grabado, vidrio, fotografía, informática- salas de usos múltiples, locales de ensayo y danza, biblioteca y otros servicios, dotados con elementos de mobiliario que en su mayor parte han sido reciclados y que incluyen piezas de maquinaria industrial procedentes de la antigua fábrica de camisas [10].

Desde el centro se trabaja con un programa de artistas en residencia, incluido dentro de la red internacional de centros y programas de residencias artísticas Res Artis. A través de la red Trans Europe Halles, TEH, los creadores pueden participar en talleres y exposiciones en otras ciudades del Arco Atlántico. Otra de sus apuestas más relevantes es el programa de soporte a la creación que comprende un conjunto de acciones que aspiran a completar la formación de los creadores, favorecer la adquisición de competencias complementarias y capacitarles para la preparación y gestión de proyectos culturales.

Entre los diferentes proyectos europeos a los que se ha asociado la Factoría Cultural es destacable Wom@rts: Women's Equal Share of Presence in the Arts and Creative Industries que, integrado en el Programa Europa Creativa de la Comisión Europea y liderado por el Concello de Santiago de Compostela está dirigido a luchar contra la desigualdad de género en el campo de las artes y la cultura ${ }^{18}$. A este puede añadirse 4HCREAT: Cuádruple hélice para estimular la innovación en las PYMEs Culturales y Creativas Atlánticas, un proyecto seleccionado por el programa Interreg Espacio Atlántico de la Comisión Europea y liderado por la universidad escocesa Glasgow Caledonian University ${ }^{19}$. A través de esta iniciativa son promovidos modelos transnacionales de transferencia de conocimiento, mejorando la aplicación de resultados de I+D de los centros de investigación en las industrias creativas y creando además nuevos productos aprovechando las oportunidades de la tecnología y de la economía digital. Entre sus actividades 4HCREAT incluye medidas de análisis de mercado y la creación de modelos de cooperación empresariales, cursos de formación, participación en ferias y promoción de acuerdos de cooperación y coproducción en ámbitos culturales. 


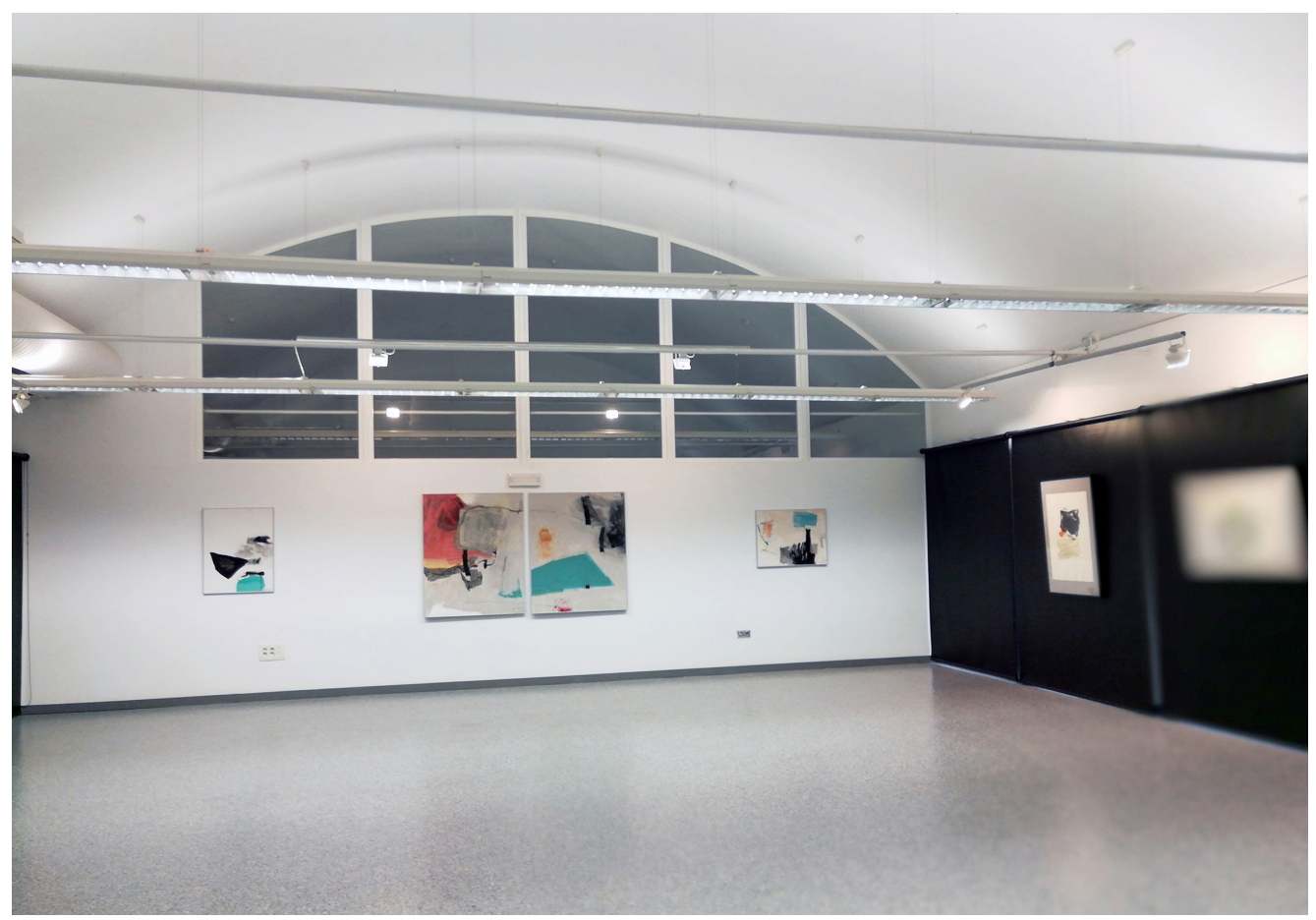

10. Sala de exposiciones, situada en la planta baja de la Factoría Cultural. Fotografía de la autora

\section{Conclusión}

Con la aspiración de transformarse en una ciudad creativa Avilés se ha volcado en la creación de una nueva identidad, abandonando sus señas distintivas, su pasado industrial, en favor de otros indicadores sustentados en la reconversión económica, la regeneración urbana, la apuesta por las actividades de servicios, la producción tecnológica y la innovación. El eje central de este cambio es un proyecto estratégico sustentado en la reconversión urbana, la regeneración de la ría y de los espacios portuarios y la implantación de una cultura basada en el fomento de la creatividad. Al amparo de este proyecto se ha diseñado y construido un gran complejo cultural, avalado por una firma de prestigio, el Centro Nie- meyer, en torno al cual se ha diseñado una marca ligada al diseño, al arte, a la cultura y a la innovación.

Dentro de este proceso, la ciudad ha impulsado un conjunto de equipamientos que, en el marco de las industrias creativas, está liderado por la Factoría Cultural: un espacio de carácter multidisciplinar, emplazado en un complejo industrial reconvertido, asociado a diversos proyectos europeos y que conjuga formación, talleres, espacios expositivos, redes de colaboración y programas de residencia para artistas.

Con todo, en Avilés se ha ido forjando una nueva identidad y el Centro Niemeyer se ha convertido en el símbolo del recambio, del nuevo Avilés postindustrial, con todas sus contradicciones, ambigüedades e indefiniciones.

\section{Notas}

1 Investigación incluida dentro del proyecto vinculado al Programa Estatal de Fomento de la Investigación Científica y Técnica de Excelencia, «Puerto y ciudad en la era postindustrial: Avilés y Bilbao, patrimonio, arquitectura y evolución urbana» (MCIU-19- PGC2018-094281-B-100).

2 La noción de sociedad postindustrial ha sido abordada desde diferentes perspectivas, orientadas hacia la economía, la sociología, la política y la filosofía. Entre otros: Bell, 1989; Brzezinski, 1970; Inglehart, 1999. 
3 La construcción de una nueva identidad para Avilés ha acarreado no pocos esfuerzos en aras de lograr su inserción en redes internacionales como Conference of Atlantic Arc Cities, International Association of Education Cities y Cool Cities.

4 Plan General de Ordenación del Término Municipal de Avilés, en Bopa, n. 163, 15 julio 2006.

5 El arquitecto Eduardo Leira (Madrid, 1944) ha sido responsable entre otros planes urbanísticos del Plan General de Madrid de 1985 y del Plan Territorial Parcial del Bilbao Metropolitano de 2006. Son constatables los paralelismos existentes en lo que al enfoque urbanístico se refiere entre los planeamientos de Avilés y Bilbao.

6 En el año 2010 Avilés obtenía la distinción de «Ciudad de la Ciencia y la Innovación» y entraba a formar parte de la Red Innpulso, Red de Ciudades de la Ciencia y la Innovación.

7 El principal escenario de las intervenciones ha sido la otrora dársena siderúrgica de la Empresa Nacional Siderúrgica S. A., Ensidesa. El desmantelamiento de la cabecera siderúrgica, avanzada la década de 1990, tuvo un marcado impacto en la ciudad de Avilés, no solo desde el punto de vista económico y social, sino también en lo que a la vertiente patrimonial se refiere con la impune destrucción, pese a la protesta de numerosos colectivos, asociaciones y ciudadanos, de buena parte de sus instalaciones.

8 El proyecto La Isla de la Innovación fue concebido por la Fundación Metrópoli, contando como socios institucionales con Infoinvest S. A., el Gobierno del Principado de Asturias, Sogepsa, el Ayuntamiento y la Autoridad Portuaria de Avilés.

9 Reconocido como el último de los arquitectos modernos, con una prolífica trayectoria profesional, el brasileño Oscar Niemeyer (1907-2012), continuador en sus inicios de la renovadora visión de Le Corbusier, ha sido galardonado entre otros con el Premio Pritzker de Arquitectura, en 1987 y el Premio Príncipe de Asturias de las Artes, en 1989. Entre la abundante bibliografía sobre el autor pueden destacarse los trabajos de Botey (1996) y Philippou (2013).

10 Equipamientos como la Biblioteca de la Universidad de Deusto, de Rafael Moneo, el Paraninfo de la Universidad del País Vasco, proyectado por Álvaro Siza, o la controvertida rehabilitación de la Alhóndiga en espacio lúdico-cultural, a cargo de Phillipe Stark, ponen de relieve el nuevo rumbo que Bilbao ha ido tomando (Esteban, 2000).

11 Especialmente relevante en este sentido es el proceso de desmantelamiento sistemático de las instalaciones de la planta de siderurgia integral de ENSIDESA, de cuyos últimos coletazos seguimos siendo testigos en la actualidad, a pesar de la inclusión del conjunto en el catálogo de referencia del Plan Nacional de Patrimonio Industrial, en el Registro del DOCOMOMO Ibérico y en los 100 elementos del Patrimonio Industrial en España (Tielve y Suárez, 2011: 144-145) a propuesta de TICCIH-España.

12 En abril de 2007 se llevaba a cabo la primera de estas actividades promocionales, «Niemeyer 100 por 100 », una exposición fotográfica desarrollada en el paseo de la Ría de Avilés que mostraba obras del arquitecto Niemeyer. En diciembre de ese mismo año, fruto del acuerdo institucional con la London School of Economics, se celebró la primera cumbre internacional del llamado C-8 -agrupación integrada por centros como el Pompidou de Paris, Lincoln Center de Nueva York, Barbican Center de Londres, Centro Cultural de Hong Kong o la Opera de Sidney- a fin de poner de relieve el potencial cultural del equipamiento.

13 Aunque no siempre ocurre así, ciertamente es indudable el impulso que algunos nuevos equipamientos culturales han dado a la dinamización turística y socioeconómica de las ciudades y los efectos positivos que han tenido en la regeneración urbana (Lorente, 2008).

14 El complejo fue erigido en 1946 a partir del proyecto elaborado por el arquitecto municipal Tomás Menéndez Abascal. La fábrica, levantada a instancias de Segundo Camino Piedra, constaba de dos naves adosadas, destinadas a sala de máquinas y almacenes. Entrada la década de 1990 la producción atravesó una grave crisis y, como resultado, el 28 de diciembre de 1995 veía clausurada su actividad.

15 Aunque no siempre se logra, es cierto que en muchos casos estos nuevos centros adquieren un importante papel en los procesos de regeneración urbana (Lorente, 2008: 60).

16 Heredero del espíritu de Bauhaus en lo que a la integración de las artes y simbiosis entre industria y creatividad se refiere, el espacio cuenta con una trayectoria dilatada y un innegable prestigio internacional. Las dotaciones de ZKM incluyen institutos y laboratorios de medios audiovisuales, música y acústica, investigación básica, medios, economía y cinematografía, junto con dos museos de arte contemporáneo y de nuevos medios.

17 El complejo fue construido en tres fases entre los años 2009 y 2012. Su financiación corrió a cargo del Ayuntamiento de Avilés, junto con el INJUVE y contando, a través del proyecto Innovate, con fondos europeos.

18 El proyecto cuenta con un presupuesto total de algo más de tres millones de euros y una subvención europea del 50\%, distribuidos entre una red de diez socios. Su desarrollo temporal arranca en octubre de 2017 y se prolonga hasta octubre de 2021.

19 Cuenta con la participación de la Glasgow Caledonian University, el Instituto Universitario de Lisboa, el parque tecnológico Laval Technopole y la institución Bordeaux Euratlantique, en Francia, así como la Escuela de Arte y Diseño de Limerick Institute of Technology, en Irlanda, además de la Factoría Cultural.

\section{Bibliografía}

ALEMANY, Joan (2015), «Incidencia del puerto en la ciudad. La experiencia latinoamericana de renovación de los waterfronts», Transporte y Territorio, n. ${ }^{\circ} 12$ (Ejemplar dedicado a Puerto y Ciudad), pp. 70-86.

BELL, Daniel (1989), El advenimiento de la sociedad post-industrial, Alianza, Madrid.

BERMEJO, María del Carmen y ÁLVAREZ, María Soledad (2018), «El Centro Cultural Internacional Oscar Niemeyer», en ÁLVAREZ, María Soledad (coord.), Focos de creación, impulso e innovación. El Centro Niemeyer, Trea, Gijón, pp. 75-140.

BOTEY, Josep María (1996), Oscar Niemeyer, Gustavo Gili, Barcelona.

BRZESINSKI, Zbigniew (1970), La era tecnotrónica, Paidós, Buenos Aires. 
COMUNIDADES EUROPEAS (1993), Libro Blanco Crecimiento, competitividad, empleo. Retos y pistas para entrar en el siglo XXI, Suplemento 6/93 del Boletín de las Comunidades Europeas, Oficina de Publicaciones Oficiales de las Comunidades Europeas, Luxemburgo.

CONSEJERÍA DE CULTURA Y TURISMO DEL PRINCIPADO DE ASTURIAS (2009), Libro Blanco sobre Industrias Creativas en el Principado de Asturias, Oviedo.

- (2010), Plan Director del Centro Niemeyer.

ESTEBAN, Marisol (2000), Bilbao, luces y sombras del titanio. El proceso de regeneración del Bilbao metropolitano, Universidad del País Vasco, Bilbao.

ESTANKONA, Andrea (2013), «Fábricas de creación, la capacidad performática de la cultura visual: imagen, imaginación e innovación en la cultura global», Ausart, n. ${ }^{\circ} 1$, pp. 25-32.

FUNDACIÓN METRÓPOLI (2008), La Isla de la Innovación, Madrid.

INGLEHART, Ronald (1999), Modernización y postmodernización, Centro de Investigaciones Sociológicas, Madrid.

LEIRA, Eduardo y CALVO, Luis, (2002-2003) «Avilés ante un nuevo futuro», Ciudades. Revista del Instituto Universitario de Urbanística de la Universidad de Valladolid, n. ${ }^{\circ}$, pp. 103-128.

LORENTE, Jesús Pedro (2008), «Los nuevos museos de arte contemporáneo en el cambio de milenio: una revisión conceptual y urbanística», Museo y Territorio, n. ${ }^{\circ} 1$, pp. 59-86.

MASBOUNGI, Ariella (2003), Nantes, la Loire déssine le projet, Editions de la Villette, Paris.

MENÉNDEZ, Rebeca (2014), «Cuatro épocas, cuatro proyectos de ciudad: la transformación urbanística de Avilés a través de los Planes Generales de Ordenación Urbana», en ÁLVAREZ, María Soledad (coord.), Espacios portuarios y villas costeras. Modelos de estrategias urbanísticas y patrimoniales de regeneración y transformación del litoral asturiano, Eikasia, Oviedo, pp. 85-113.

PHILIPPOU, Styliane (2013), «El modernismo radical de Oscar Niemeyer», Arquitectura y Urbanismo, vol. XXXIV, n. ${ }^{\circ}$ 2, agosto, pp. 5-26.

SARRIUGARTE, Iñigo (2014), «Del Proyecto Guggenheim a la revitalización del Zorrotzaurre: regeneración del tejido urbano y cultural del Gran Bilbao", Ábaco, vol. 2/3, n. ${ }^{\circ} 80 / 81$, pp. 50-51.

SCOTT, Allen. J. (2006), "Creative Cities: Conceptual Issues and Policy Questions», Journal of Urban Affairs, n. ${ }^{\circ} 28$, pp. 1-17.

SUÁREZ, Faustino (2013), «Los instrumentos de protección del Patrimonio Industrial en el marco del planeamiento y la ordenación urbanística», en RUIZ DE LA PEÑA, D. (coord.), Los Catálogos Urbanísticos en el Principado de Asturias, Servicio de Publicaciones de la Universidad de Oviedo, Oviedo, pp. 187-213.

TIELVE, Natalia (2016), «La memoria del pasado industrial: conservación, reutilización y creación de nuevos equipamientos», E-rph: Revista electrónica de Patrimonio Histórico, n. ${ }^{\circ}$ 19, pp. 72-99.

- (2018), «El Centro Niemeyer y la promoción de nuevos equipamientos: industrias creativas, innovación y desarrollo», en ÁLVAREZ, Soledad (coord.), Focos de creación, impulso e innovación: El Centro Niemeyer, Trea, Gijón, pp. 141-214.

TIELVE, Natalia y SUÁREZ, Faustino (2011), "Conjunto industrial de ENSIDESA», en W. AA., 100 elementos del Patrimonio Industrial en España, Instituto de Patrimonio Cultural de España, TICCIH-España, Madrid, pp. 144-145. 